\title{
Development of a Mathematical Model for Kinetics of Obtaining Isocyanate via a Non-Phosgene Method for Example Benzylisocyanate
}

\author{
Dashkin R. R. ${ }^{1}$, Shishanov M. V. ${ }^{2}$ \\ ${ }^{1}$ Head of the MUCTR Engineering center, D. Mendeleev University of Chemical Technology of Russia, Moscow, Russia, 20 Geroyev Panfilovtsev str., \\ Moscow \\ ${ }^{2}$ Leading engineer of the MUCTR Engineering center, D. Mendeleev University of Chemical Technology of Russia, Moscow, Russia, 20 Geroyev \\ Panfilovtsev str., Moscow
}

Received: 02/07/2020

Accepted: 08/10/2020

Published: 20/12/2020

\begin{abstract}
The paper considers the process of developing a mathematical model for kinetics of obtaining isocyanates via a non-phosgene method - non-catalytic thermolysis of carbamates. A mathematical model is presented, kinetic parameters such as activation energy and a preexponential multiplier are ascertained.
\end{abstract}

Keywords: Isocyanate production, non-phosgene technology, mathematical model, thermolysis of carbamates, kinetics of decomposition

\section{Introduction}

Isocyanates are one of the most relevant products of the chemical industry, as they are primary products for the polyurethanes production, which are used in construction, in the production of automotive parts, insulation materials, paintwork products, adhesives, paints, fillers for upholstered furniture (1). The main method for obtaining polyurethanes is the interaction of isocyanates by nucleophilic addition of polyols (2). In addition, isocyanates are valuable intermediates in organic synthesis of pesticides and other biologically active substances (3). Despite the wide range of applications of isocyanates in the world practice, the main method of production is still the technology using phosgene (4-7), which makes production unecological, and modern standards and requirements for the protection of human health and the environment leads to an urgent need to develop an environmentally friendly method for obtaining isocyanates.

It is known that esters of N-substituted carbamic acid can be precursors in the synthesis of isocyanates. The method of obtaining isocyanates $(8,9)$, which consists in splitting alcohol from carbamate when heated or with various catalysts (Fig. 1), can be called one of the most promising.<smiles></smiles>

Figure 1: The general scheme of the carbamates decomposition reaction

The complexity of implementing this method in industry is related to the reversibility of the reaction. However, there are ways to shift the balance towards product formation by reducing the concentration of one of the products. So in practice, a promising solution to this problem can be the rectification of a products mixture, as a result of which the alcohol will be removed from the resulting mixture. Thus, the equilibrium of the decomposition reaction will shift towards the formation of the target isocyanate, ensuring a satisfactory yield of the product. For today, the process of obtaining isocyanates by decomposing carbamates remains poorly understood, in particular, the kinetic data of the reaction are almost absent. In the conditions of growing demand for isocyanate raw materials both in Russia and on the world market, as well as the lack of industrially applicable phosphorless technology for producing isocyanates, the study of the method for obtaining isocyanates by thermal decomposition is an extremely relevant area of research. Due to the need to study the kinetics of the carbamates decomposition, a mathematical model for the kinetics of the obtaining benzylisocyanate reaction was constructed with the determination of kinetic parameters - the pre-exponential multiplier and the activation energy $\left(\mathrm{k}_{0}\right.$ and $\left.\mathrm{E}_{\mathrm{A}}\right)$ from experimental data. The mathematical model will allow to scale the process and work out critical parameters at the design stage for a semi-industrial plant with non-isothermal conditions in the reactor.

\subsection{Experimental part}

The mathematical model was constructed using experimental data obtained using a plant for thermal decomposition of carbamates. To develop the kinetic model, we conducted a series of experiments using an experimental setup, the flow diagram of which is shown in figure 2 . The Decomposed carbamate was pre-mixed with an inert gas (argon) and the mixture was heated to a predetermined temperature in unit 1 . Further, the mixture was delivered in gaseous form to the unit 2 (reactor), where the process of carbamate decomposition took place and the formation of a products mixture with target isocyanate and alcohol. Then the reaction products were fed to the sorption unit 3 , where the sorption solution was located. In this unit, the product of the carbamate decomposition reaction (isocyanate) reacted with $\mathrm{N}$ methylbenzylamine to form urea, and the inert carrier gas partially together with alcohol went into the waste gases. 


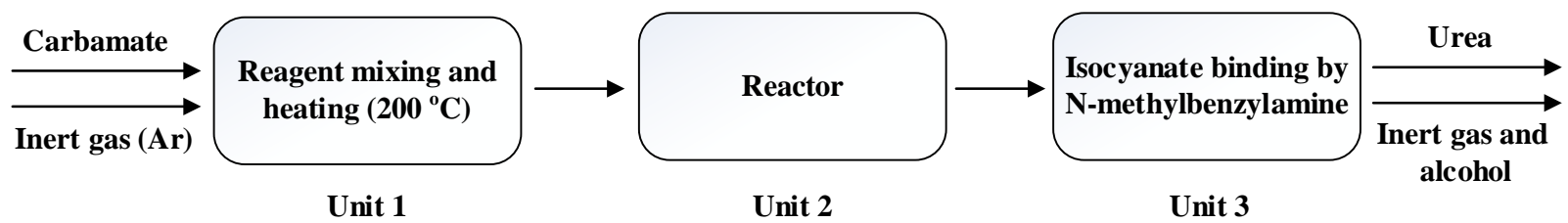

Figure 2: The block diagram of the experimental plant of carbamate thermolysis

The inert gas in the carbonate decomposition was used to reduce the partial pressure in order to shift the equilibrium of the reaction towards the products. In addition, this method was able to reduce the vapor pressure of carbamate, which reduces its boiling point. This made it possible to conduct experiments in temperature ranges which were convenient and implemented in practice. The transformation of a substance into a gas phase in the heating unit is necessary to separate the processes of evaporation and decomposition in space, which allowed us to more accurately study the features of the decomposition reaction. The reaction was performed in a flow-through tubetype reactor. A sketch of the reactor used is shown in figure 3 , indicating the dimensions and the designation of the inlet and outlet holes. The choice of this type of reactor is determined by the type of chemical reaction and the ability to conduct the process continuously.

The reactor was heated using an electric heating element located on the outer surface of the metal tube of the reactor. In this case, the temperature profile inside the reactor cannot be set constant along the length of the entire reactor, because there are zones of heating the gas phase entering the reactor and zones of cooling the reactor to the external environment at its ends. In addition, the temperature profile along the length of the reactor depends on the maximum temperature in the middle of the reactor and velocity of the carrier gas (Fig. 4).

\subsection{A mathematical model for the kinetics of carbamates thermal decomposition}

To solve the problem of modeling the decomposition of carbamate, the necessary calculations of the material and heat balances were performed. The following conditions were used for the permanent section tube reactor:

1. The operating mode must ensure uniformity of parameters across the reactor cross-section, this is provided either by the turbulent gas flow mode (high flow rates) or by using distribution nozzles inside the reactor;

2. The ratio of the reactor length to the cross-section diameter 1/D must be more than ten units (in our case, the ratio is 18).
If the above conditions are met, the effect of reverse mixing can be ignored, since the temperature and concentration of the reaction mixture components do not change along the crosssection of the reactor. The hydrodynamic mode of the experimental reactor was determined by the abrupt introduction of the initial carbamate into it without external heating as a tracer (i.e., by the step perturbation method). Based on the measurement of its concentration at the reactor outlet, the system response curves were obtained (Fig. 5).

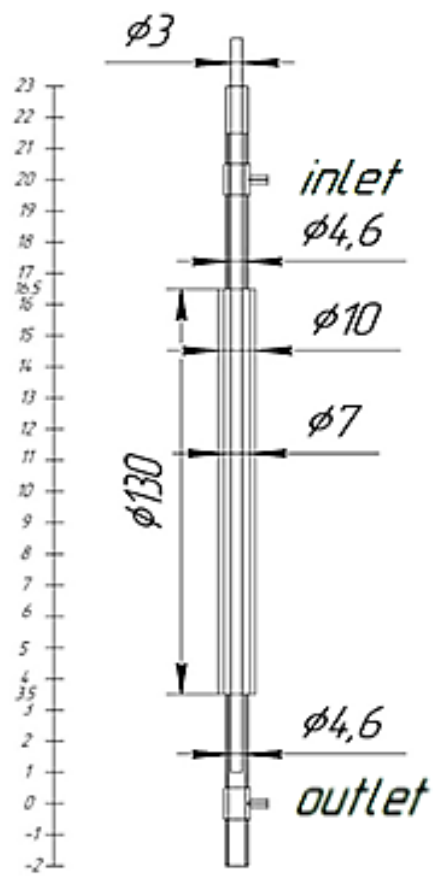

Figure 3: Sketch of an experimental reactor.

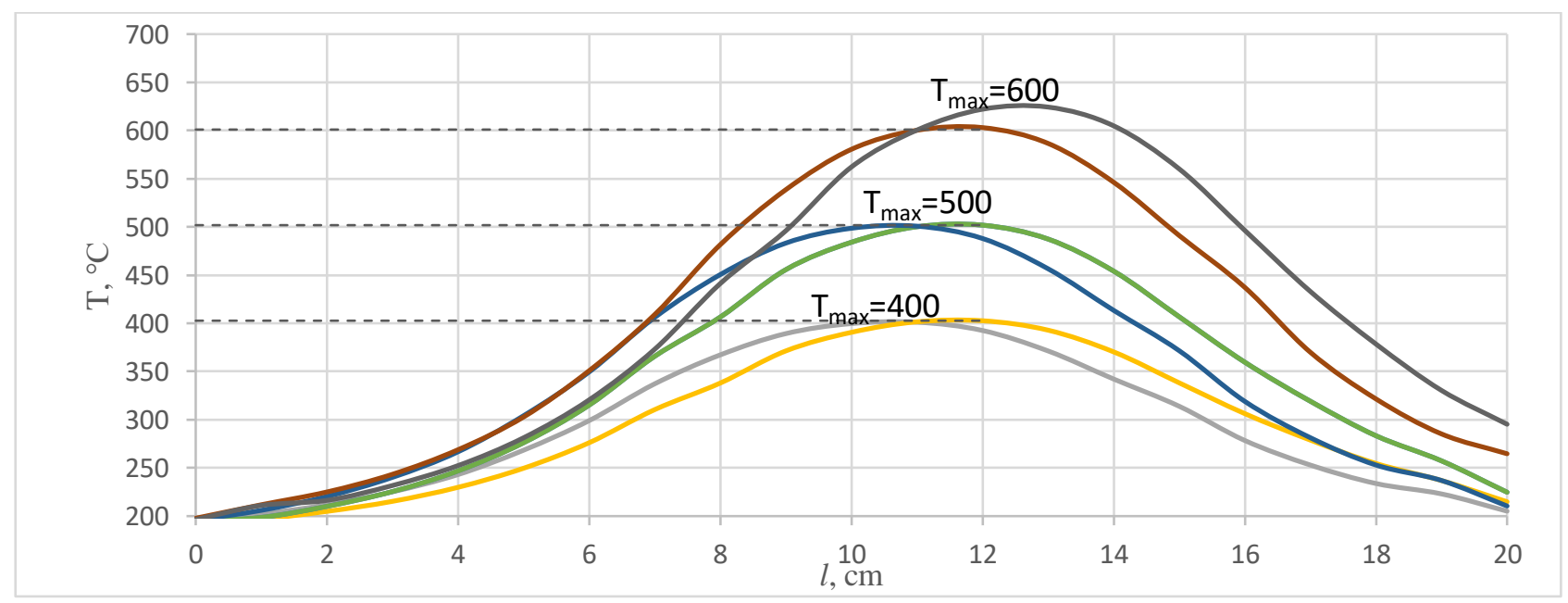

Figure 4: Profiles of temperature along the length of reactor at different temperatures and the carrier gas velocity. 


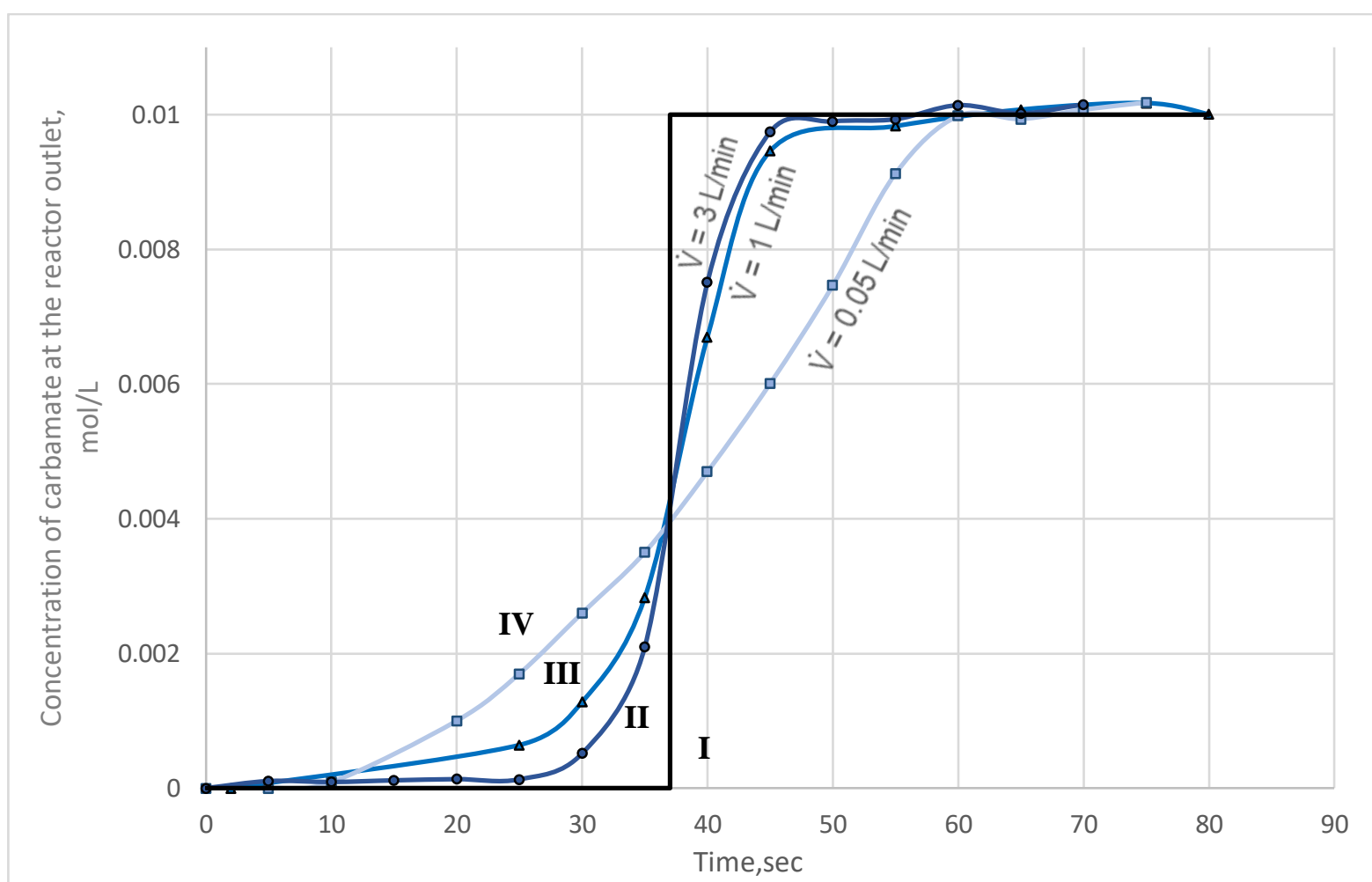

Figure 5: F-curves of response for the ideal displacement reactor (I) and the investigated reactor at different speeds of the carrier gas supply (II-IV)

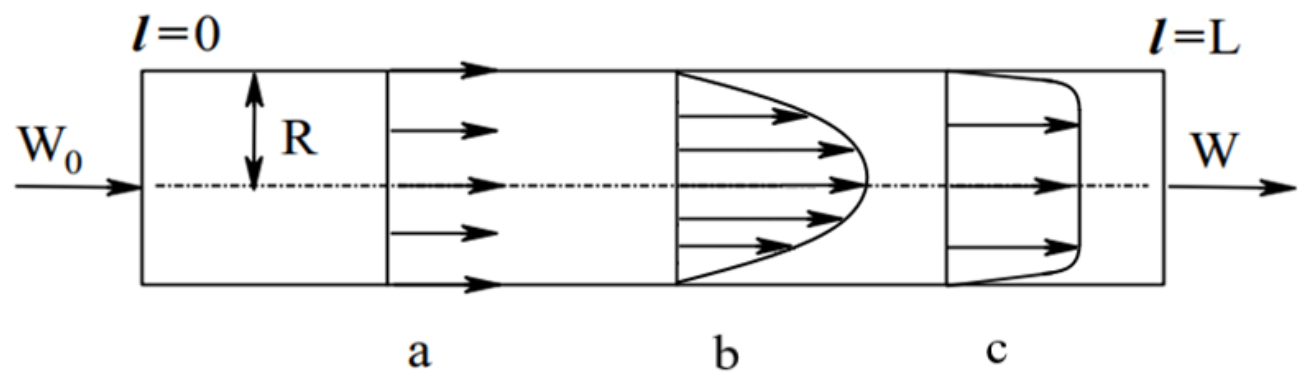

Figure 6: Diagram of flow modes in the reactor ( $\mathrm{a}$ - an ideal displacement reactor, $\mathrm{b}-\mathrm{a}$ laminar flow mode, $\mathrm{c}-\mathrm{a}$ turbulent flow mode)

The logarithmic nature of the obtained graph allows us to conclude that the most suitable model for describing the reactor used is the ideal displacement reactor (Fig. 6). Therefore, this model was used for further calculations for the tube reactor used.

\subsection{Material balance for a perfect displacement reactor}

Schematically the decomposition reaction of the investigated carbamate can be represented as follows:

$$
\mathrm{A} \rightleftarrows \mathrm{B}+\mathrm{C}
$$

A - N-benzyl-O-methylcarbamate,

B - benzylisocyanate, $\mathbf{C}$ - methanol

The general equation of a non-stationary mass transfer with sources (10):

$\frac{\partial c_{j}}{\partial \tau}+\operatorname{div}\left(c_{j} \cdot w\right)=\sum_{i=1}^{p} v_{i j} \cdot r_{i}+\operatorname{div}\left(D_{j} \operatorname{grad} c_{j}\right)$ where $c_{j}$ is the concentration of the $j$-th product, mol/L; $\tau_{\mathrm{i}}$ is astronomical time of the process, sec; $w$ is linear flow rate, $\mathrm{m} / \mathrm{sec} ; v_{\mathrm{ij}}$ is stoichiometric coefficient of the substance in the reaction; $r_{i}$ is invariant speed of the $i$-th stage of the process, $\mathrm{mol} / \mathrm{sec}$; and $D_{j}$ is diffusion coefficient of the substance, $\mathrm{m}^{2} / \mathrm{sec}$. In the framework of the model of an ideal displacement reactor, it can be assumed that there is no transfer of the substance by diffusion and reverse mixing $\left(\operatorname{div}\left(D_{j} \operatorname{grad} c_{j}\right)=0\right)$. Thus, the change in the concentration of the substance $c_{j}$ occurs only in the coordinate $l$, and the material balance equation will have the form:

$$
\frac{\partial c_{j}}{\partial \tau}+\frac{\partial\left(c_{j} \cdot w\right)}{\partial l}=\sum_{i=1}^{p} v_{i j} \cdot r_{i}
$$

In the course of experiments, the steady temperature profile, the carrier gas flow rate and the ratio of reagents are maintained, and the data obtained after the process enters stationary mode are taken into account when processing the results, so we can assume $\frac{\partial c_{j}}{\partial \tau}=0$, then:

$\frac{d\left(c_{j} \cdot w\right)}{d l}=\sum_{i=1}^{p} v_{i j} \cdot r_{i}$ 
Assuming that $c_{j}$ is the concentration of component $\mathbf{A}$ $C_{A}=\frac{N_{A}}{\dot{V}}$

where где $N_{A}$ is the mole flow of component $\mathrm{A}, \mathrm{mol} / \mathrm{sec}$ and $\dot{V}$ is volume flow, $\mathrm{L} / \mathrm{sec}$. Hence, the material balance for a simple reaction with varying volume:

$\frac{d\left(w \cdot C_{A}\right)}{d l}=-v_{A} \cdot r$ A:

The equation for the rate of chemical reaction for substance

$r_{A}=v_{A} \cdot r=k \cdot\left(C_{A}\right)^{m}$

where $m$ is reaction order and substitute in (1.3)

$\frac{d}{d l}\left(w \cdot \frac{N_{A}}{\dot{V}}\right)=-k \cdot\left(C_{A}\right)^{m}$

where $\frac{V}{w}=S$ is a cross sectional area.

$\frac{1}{S} \frac{d N_{A}}{d l}=-k \cdot\left(C_{A}\right)^{m}$

Under process conditions, the volume flow depends on temperature and can be expressed using the ideal gas law with a correction compressibility coefficient, since $N_{I} \gg N_{A}$ (the ratio $\frac{N_{I}}{N_{A}}$ in experimental conditions from 29 to 1760). This allowed us to get the function of the volume flow from the temperature.

$\dot{V}=\frac{N_{\text {total }} \cdot R \cdot T}{Z \cdot P_{\text {total }}}=\frac{N_{I} \cdot R \cdot T}{Z \cdot P_{\text {total }}}$

where $Z$ is a compressibility factor, $Z$ is equal of 1 for incompressible gas; $N_{I}$ is the mole flow rate of the carrier gas, $\mathrm{mol} / \mathrm{sec} ; R$ is the gas constant; and $P_{\text {total }}$ is the pressure in the reactor, $\mathrm{Pa}$. The concentration of component $\mathrm{A}$ in terms of molar flows and temperature at a given temperature profile along the length of the reactor, depending on the velocity of the carrier gas:

$C_{A}=\frac{N_{A}}{V}=\frac{N_{A} \cdot P_{t o t a l}}{N_{I} \cdot R \cdot T(l)}$

The dependence of the reaction rate constant on the temperature according to the Arrhenius equation:

$k=k_{0} \cdot \exp \left(-\frac{E}{R T(l)}\right)$

The molar flow of a substance in terms of the degree of transformation:

$N_{A}=N_{A_{0}} \cdot\left(1-X_{A}\right)$

Substituting the obtained expressions (2.2), (2.3) and (2.4) in equation (1.7), we get:

$\frac{1}{S} \cdot \frac{d}{d l}\left(N_{A_{0}} \cdot\left(1-X_{A}\right)\right)=-\left(k_{0} \cdot \exp \left(-\frac{E}{R T(l)}\right)\right)$.

$\left(\frac{\left(N_{A_{0}} \cdot\left(1-X_{A}\right)\right) \cdot P_{\text {total }}}{N_{I} \cdot R \cdot T(l)}\right)^{m}$

Open the brackets and make the equation clear: $\frac{d}{d l} X_{A}=S \cdot\left(N_{A_{0}}\right)^{m-1} \cdot k_{0} \cdot \exp \left(-\frac{E}{R T(l)}\right) \cdot\left(\frac{P_{\text {total }}}{N_{I} \cdot R \cdot T(l)}\right)^{m}$.

$\left(1-X_{A}\right)^{m}$

Thus, we obtained a differential dependence of the degree of transformation of substance A along the length of the reactor, taking into account the molar flow of the inert gas, the molar flow of substance $\mathrm{A}$ at the entrance to the reactor and the temperature profile.

\subsection{Thermal balance for a perfect displacement reactor}

Due to the fact that the temperature profile along the length of the reactor is ascertained and it does not depend on the flow of a chemical reaction inside the reactor, since the reaction is performed at high dilution, there is no need to compile a heat balance. To mathematically describe the temperature profile along the length of the reactor at different carrier gas flows, a polynomial function of the temperature of the reactor length and the volume flow of the inert gas is constructed (2.7). Then the resulting polynomial is substituted in (2.6).

$T=T(l, N)$

\subsection{Determination of kinetic parameters based on experimental data}

In equation (2.6), $E$ (an activation energy), $k_{0}$ (a preexponential multiplier) and $m$ (the reaction order) remain unknown. These parameters are unique for each carbamate. In this paper, the kinetics of $\mathrm{N}$-benzyl- $\mathrm{O}$-methylcarbamate decomposition at different temperature profiles is considered. Due to the fact that the decomposition reaction of carbamate is reversible, to determine the concentration of the formed benzylisocyanate at the exit from the reactor, it is bound by interaction with a secondary amine (methylbenzylamine), which leads to the urea formation.<smiles>[R]NC(=O)N(C)Cc1ccccc1</smiles>

Figure 7: Reaction of the urea formation in the sorption unit

A sample was taken from the resulting solution, and the concentration of urea and unreacted carbamate was determined using the HPLC method. Based on the results of the analysis, the degree of carbamate transformation was calculated for a series of experiments with different volume flow of an inert gas and at different temperature profiles. Time, when the mixture was in the reactor, was derived from the ideal gas law equation, representing the internal volume of the reactor as the composition of the cross-section area by the length $(d V=$ $S d L)$ :

$\frac{S d l}{d \tau}=\frac{N_{I} R T(l)}{P_{\text {total }}}$

After separating the variables and integrating both parts of the equation, we get:

$\int_{0}^{L} \frac{d l}{T(l)}=\frac{N_{I} R}{S P_{\text {total }}} \int_{0}^{\tau} d \tau$

Then, the residence time of the mixture in the reactor is $\tau$, which will depend on the temperature function along the length of the reactor. 
Table 1: Dependence of the transformation degree on the temperature mode of the reactor

\begin{tabular}{|c|c|c|c|c|}
\hline № & $\begin{array}{l}\text { Maximum temperature in the } \\
\text { reactor, } \mathrm{T}_{\text {reactor }}\left({ }^{\circ} \mathrm{C}\right)\end{array}$ & $\begin{array}{l}\text { The molar flow of the carrier } \\
\text { gas, } \mathrm{N}(\mathrm{mol} / \mathrm{sec})\end{array}$ & $\begin{array}{l}\text { Time when the mixture was in the } \\
\text { reactor, } \tau(\mathrm{sec})\end{array}$ & $\begin{array}{c}\text { Degree of } \\
\text { transformation, } X\end{array}$ \\
\hline 1 & 250 & 0,08 & 1,880 & 0,664 \\
\hline 2 & 300 & 0,15 & 0,948 & 0,547 \\
\hline 3 & 350 & 0,25 & 0,540 & 0,691 \\
\hline 4 & 350 & 0,05 & 2,703 & 0,878 \\
\hline 5 & 400 & 0,4 & 0,322 & 0,589 \\
\hline 6 & 400 & 0,05 & 2,577 & 0,897 \\
\hline 7 & 450 & 0,75 & 0,164 & 0,791 \\
\hline 8 & 450 & 0,05 & 2,577 & 0,923 \\
\hline 9 & 500 & 1,50 & 0,078 & 0,732 \\
\hline 10 & 500 & 0,05 & 2,363 & 0,964 \\
\hline 11 & 550 & 2,50 & 0,045 & 0,611 \\
\hline 12 & 550 & 0,05 & 2,272 & 0,987 \\
\hline 13 & 600 & 3,00 & 0,036 & 0,755 \\
\hline 14 & 600 & 2,5 & 0,043 & 0,797 \\
\hline 15 & 600 & 2,00 & 0,054 & 0,962 \\
\hline 16 & 600 & 1,5 & 0,072 & 0,786 \\
\hline 17 & 600 & 1,00 & 0,109 & 0,901 \\
\hline 18 & 600 & 0,75 & 0,145 & 0,923 \\
\hline 19 & 600 & 0,50 & 0,218 & 0,948 \\
\hline 20 & 600 & 0,40 & 0,273 & 0,965 \\
\hline 21 & 600 & 0,30 & 0,364 & 0,979 \\
\hline 22 & 600 & 0,20 & 0,546 & 0,985 \\
\hline 23 & 600 & 0,15 & 0,729 & 0,991 \\
\hline 24 & 600 & 0,08 & 1,367 & 0,998 \\
\hline 25 & 600 & 0,05 & 2,188 & 0,994 \\
\hline
\end{tabular}

$\tau=\frac{S P_{\text {total }}}{N_{I} R} \int_{0}^{L} \frac{d l}{T(l)}$

As a result, the degree of transformation data was obtained. This conversion rate corresponds to the conversion rate at the end of the reactor:

$X_{\text {fin. exp. }}=X_{(\text {f fin. })}$

To determine the kinetic parameters, it is necessary to represent the degree of transformation of $X_{\mathrm{A}}$ as a finitedifference approximation by means of Taylor series expansion. To solve this problem, it is necessary to represent the length of the reactor as a set of $\mathrm{n}$ segments of finite length and to determine the degree of transformation $X_{A_{i}}$ on each segment. In this case, as a result of numerical integration of a nonlinear differential equation with the parameter:
$X_{A_{i+1}}=X_{A_{i}}+\left(S \cdot\left(N_{\mathrm{A}_{0}}\right)^{m-1} \cdot k_{0} \cdot \exp \left(-\frac{E \cdot 10^{3}}{R \cdot\left(T\left(100 \cdot l_{l}\right)+273\right)}\right) \cdot\right.$

$\left.\left(\frac{P_{\text {total }}}{N_{I} \cdot R \cdot\left(T\left(100 \cdot l_{i}\right)+273\right)}\right)^{m} \cdot\left(1-X_{A}\right)^{m}\right) \cdot h$

$i=0 . . n-1$

where $h=\frac{L}{n}$ and $X_{0}=0$. Assuming that the order of the carbamate decomposition reaction is $m=1$, it is possible to find the remaining kinetic parameters by determining the minimum deviation of the function from the experimental data. Data for other orders of reaction $m$ are not included due to the inadequacy of the data obtained. For this purpose, the minimization function (3.3) was established, which allows to find the values of $E_{A}$ and $k_{0}$ using the gradient descent method based on experimental data (the reactor temperature profile, the carrier gas flow, and the degree of transformation). A difference scheme is an explicit Euler scheme. 


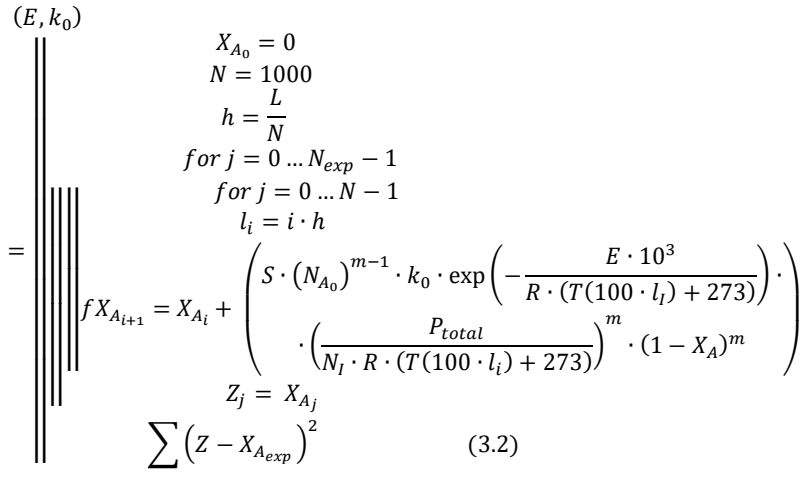

As a minimization criterion in this function, the quadratic loss function of two vectors was used: the vector of the transformation degree for experimental data and the vector of calculated data. As a result, the following values are obtained:

\begin{tabular}{|c|c|c|}
\hline Experiment \# & $X_{\text {mod }}$ & $X_{\text {exp }}$ \\
\hline 1 & 0,651 & 0,664 \\
\hline 2 & 0,588 & 0,547 \\
\hline 3 & 0,703 & 0,691 \\
\hline 4 & 0,863 & 0,878 \\
\hline 5 & 0,693 & 0,589 \\
\hline 6 & 0,863 & 0,897 \\
\hline 7 & 0,692 & 0,791 \\
\hline 8 & 0,899 & 0,923 \\
\hline 9 & 0,641 & 0,732 \\
\hline 10 & 0,963 & 0,964 \\
\hline 11 & 0,638 & 0,611 \\
\hline 12 & 0,951 & 0,987 \\
\hline 13 & 0,731 & 0,755 \\
\hline 14 & 0,787 & 0,797 \\
\hline 15 & 0,988 & 0,962 \\
\hline 16 & 0,92 & 0,786 \\
\hline 17 & 0,977 & 0,901 \\
\hline 18 & 0,925 & 0,923 \\
\hline 19 & 0,933 & 0,948 \\
\hline 20 & 0,966 & 0,965 \\
\hline 21 & 0,988 & 0,979 \\
\hline 22 & 0,992 & 0,985 \\
\hline 23 & 0,995 & 0,991 \\
\hline 24 & 0,999 & 0,998 \\
\hline 25 & 1 & 0,994 \\
\hline
\end{tabular}

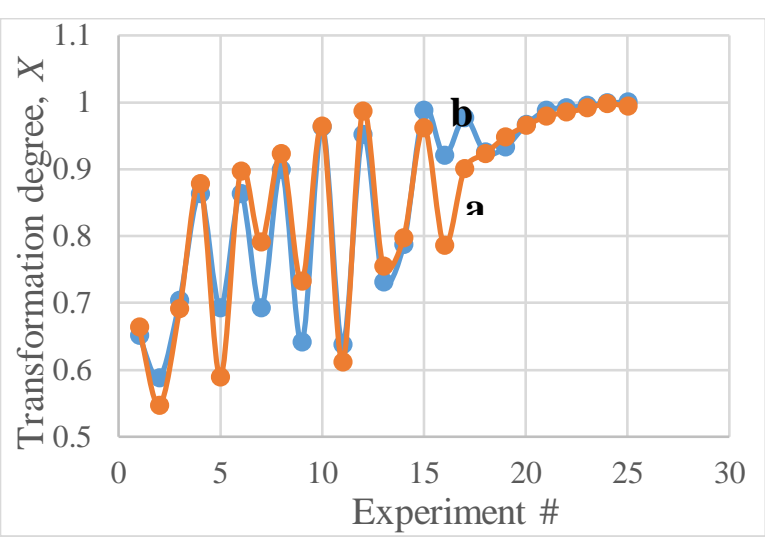

Figure 8: Graphical comparison of the experimentally obtained values (a) of transformation degrees and calculated using the constructed model (b)
The error of the obtained data was estimated using the mean square error (MSE) (3.5) and was 0.009 .

$M S E=\frac{1}{m} \sum_{i=1}^{m}\left(X_{\text {exp }}-X_{\text {mod }}\right)^{2}$

Thus, the values of $E_{\mathrm{a}}=54.22 \mathrm{~kJ} / \mathrm{mol}$ and $k_{0}=1.84 \cdot 10^{5}$ were obtained. The reaction rate constant was calculated from the Arrhenius equation.

\section{Conclusion}

During the work, kinetic data for the thermal decomposition of $N$-benzyl- $O$-methylcarbamate were obtained. Good convergence of the results indicates that the system of differential equations describes the process of carbamate decomposition quite accurately. Based on the results a mathematical model was developed. It allows to carry out engineering calculations of reactors for obtaining isocyanates by non-phosgene method. For the reaction of thermal decomposition of $\mathrm{N}$-benzyl- $O$-methylcarbamate, the values of the activation energy and the pre-exponential multiplier were obtained: $E_{\text {акт }}=54.22 \mathrm{~kJ} / \mathrm{mol}$ and $k_{0}=1.84 \cdot 10^{5}$.

\section{Acknowledgment}

Scientific work with the support of the ministry of science and higher education of the russian federation - federal target program no. $075-15-2019-1856$ of 12/03/19 (unique project identifier rfmefi60719x0315).

\section{Ethical issue}

Authors are aware of, and comply with, best practice in publication ethics specifically with regard to authorship (avoidance of guest authorship), dual submission, manipulation of figures, competing interests and compliance with policies on research ethics. Authors adhere to publication requirements that submitted work is original and has not been published elsewhere in any language.

\section{Competing interests}

The authors declare that there is no conflict of interest that would prejudice the impartiality of this scientific work.

\section{Authors' contribution}

All authors of this study have a complete contribution for data collection, data analyses and manuscript writing.

\section{References}

1. Bukharkina TV, Verzhichinskaya SV, Digurov NG, Kozlovsky R A Fundamentals of design and calculation of devices for chemical technology of fuel and carbon materials. M: MUCTR, 2015: 136 p.

2. Dai Y, Wang Y. Yao J, Wang Q, Liu L. Phosgene-Free synthsis of phenyl isocyanate by catalytic decomposition of methyl N-phe; 2008;123: 307-316.

3. Fukuoka S, Chono M, Kohno M. Isocyanate without phosgene, 1984;14: 670-676.

4. Gorbatenko V I, Zhuravlev EZ, Samarai LI. Isocyanates. Methods of synthesis and physical and chemical properties of alkyl-, aryland heterylisocyanates. K., 1987: 444 p.

5. Lebedev, AV. Organosilicon synthesis of isocyanates: II Synthesis of aliphatic, carbocyclic, and fatty-aromatic isocyanates / A.V. Lebedev et al. Russ. J. Gen. Chem. 2006;76(3):. 469-477.

6. Nagy L, Nagy T, Kuki Á, Purgel M, Zsuga M, Kéki S. Kinetics of Uncatalyzed Reactions of 2, 4'-and 4, 4'-DiphenylmethaneDiisocyanate with Primary and Secondary Alcohols. International Journal of Chemical Kinetics. 2017 Sep;49(9):643-55. 
7. Patent US 20040267047A1 Method for production of Isocyanates 2001

8. Patent US 200702620070265465A1 Process for the production of isocyanates 2007

9. Patent US 7547801 Int. Cl. C07C263/10. Process for the continuous preparation of isocyanates / F. Pohl et al. - Claimed: 26.06.2006, published:16.06.2009.

10. Saunders D, Frisch K. Chemistry of polyurethanes. Moscow: Chemistry, 1968: 87 p. 